\section{Prevention of spread of echovirus 6 in a special care baby unit}

Sir,

Carolane and colleagues describe measures aimed at preventing the spread of enterovirus infection after a fatal case of disseminated echovirus 6 infection. ${ }^{1}$ Like others they gave human immunoglobulins to all babies and closed their neonatal unit to outside referrals. ${ }^{2}$ Human immunoglobulins are not necessarily without risk. ${ }^{3}$ Our main concern, however, is the impact of closing regional units and the rationale behind this policy.

Selective reporting of severe cases will exaggerate the gravity of enterovirus infections. The risk of an epidemic ${ }^{2}$ must be weighed against the effects of closing referral units. Nursery epidemics usually occur after mother to infant-that is, vertical-transmission; the policy advocated can only interrupt horizontal transmission. Severity of infection will partly depend on the organism's virulence and inoculum size. It is not clear from previous reports how often enteroviruses fail to spread to other babies or cause only mild illness.

In the last year three babies in this unit have had enterovirus infections. We did not change admission policy nor administer human immunoglobulins. Stools and nasopharyngeal aspirates were cultured from all babies on the unit and from symptomatic staff. We found no evidence of transmission from two infants with perinatally acquired infection: a baby with fatal disseminated echovirus 7 infection nursed in the intensive care room for nine days and a baby with Coxsackie B5 meningitis nursed in a cubicle. Echovirus 9 was grown from the stools of a two month old girl with gastroenteritis and, one week later, from nasal secretions of a baby with upper respiratory infection and stools from an asymptomatic infant.

We do not think that available evidence justifies a blanket policy of closing regional neonatal intensive care nurseries to outside referrals each time an enterovirus infection is diagnosed. If our experience is representative, busy regional neonatal centres would need to be closed several times a year for lengthy periods, almost certainly leading to an increase in morbidity and mortality of infants in district units.

D IsAacs, A WiLkinson, AND E R Moxon John Radcliffe Hospital, Oxford $O X 39 D Y$

\section{Dr Carolane and co-workers comment:}

We would agree that sporadic single cases of enterovirus infection, particularly in the absence of a known virulent virus such as echo 11 in the community, do not warrant the extreme measures we have described.

In the interest of brevity, when submitting our paper, we omitted some details of the sequence of events that led to our decision on closure and hope that the Figure will clarify the situation.

On first clinical suspicion of an echovirus infection we took measures to isolate the infant, thus preventing

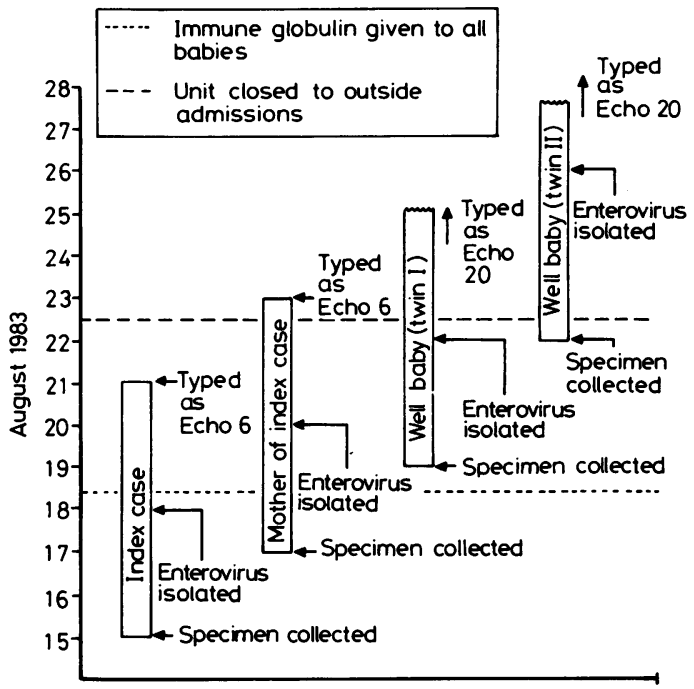

Figure Sequence of events leading to closure of neonatal unit.

horizontal transmission. The immediate contacts of the infant were also isolated, and stool specimens were collected from all the babies on the unit. Our first impression, in view of the severity of infection, was that this was likely to be an echo 11 infection, and together with past experience here as well as in Cambridge, the immediate concern was that of preventing an outbreak in the unit.

When the virus was typed as echo 6 , we had already isolated a further enterovirus from a healthy infant on the unit who had never been in contact with the sick infant, and it was this second virus isolation that concerned us that there might be spread within the unit. It was on this basis that we took action to prevent admissions. We subsequently discovered that the second baby, together with his twin brother, was infected with echovirus 20 , which caused no clinical problem.

With regard to the use of intramuscular normal immunoglobulin, we sought the advice of Dr J Nagington, who has had experience of the Cambridge echo 11 outbreaks, and as we suspected this virus at the time, we felt we must move swiftly to contain any possible outbreak.

Isaacs and colleagues mention the risk of using immunoglobulin ${ }^{3}$ but Lever et al injected their agammaglobulinaemic patients intravenously, necessitating a slight modification in the preparation of a safe intramuscular product. ${ }^{4}$ Furthermore, the biochemical criteria on which the diagnosis of non-A, non-B hepatitis in Lever's series was made have been criticised. ${ }^{5}$ We do not know of any infectious sequel after the intramuscular administration of normal human immunoglobulin made by the British Blood Products Laboratory or the Scottish Blood Transfusion Service.

In conclusion, therefore, we would agree with Isaacs and colleagues that, in retrospect, our action in closing our unit was somewhat precipitate, but at the time, with the facts 